\title{
Qualitative role of endobronchial elastography with endobronchial ultrasound in differentiating malignant and benign lesions: a retrospective single-center study from India Rajesh Gupta, Sharad Joshi, Ankit Bhatia, Nitesh Tayal, Praveen Pandey
}

Background Endobronchial ultrasound (EBUS) is useful in guiding needle biopsy of mediastinal lesions.

Sonoelastography in latest generation machines have enabled mapping the elasticity of the lymph nodes (LNs) leading to potentially better guidance in taking samples.

Aim We aim to assess the practical usefulness of elastography in assessing mediastinal lymphadenopathy by comparing the patterns seen on EBUS elastography with the final pathological diagnosis from EBUS-guided transbronchial needle aspiration.

\begin{abstract}
Patients and methods The EBUS scope (convex) was intubated via the oral route, and images were generated to evaluate the patterns produced during elastography according to color distribution: type 1 was taken as predominantly nonblue (yellow, red, and green); type 2 was less than $50 \%$ blue color, part nonblue (yellow, red, and green); type 3 predominant blue. Elastography patterns were subsequently compared with the eventual pathological results.
\end{abstract}

Results In the study period, 105 LNs in 80 patients were studied, 79 were found to be of benign nature while 26 patients were diagnosed as malignancy. Type $1 \mathrm{LNs}$ were of benign pathology in 42/46 (91.30\%) cases and malignant in 4/ $46(8.70 \%)$ cases; for type 2 LNs, $24 / 30(80 \%)$ cases were benign and $6 / 30(20 \%)$ cases were malignant. Type 3 nodes were found to be of benign nature in $13 / 29$ (44.82\%) cases

\section{Introduction}

Endobronchial ultrasound (EBUS) has gained a lot of importance in recent years and has been an integral part of the diagnostic armamentarium in the evaluation of mediastinal lymphadenopathy. It has expanded its scope from a mere diagnostic procedure to even therapeutic lymph node (LN) decompressions and implanting fiducial markers [1,2]. Lung cancer management requires careful mediastinal LN evaluation. EBUS and endoscopic ultrasound are complementary methods as in combination virtually all mediastinal and hilar nodal stations can be visualized and sampled. Selection of $\mathrm{LNs}$ to be sampled is a crucial step, affecting diagnostic accuracy and preventing undue sampling.

Elastography provided to the new generation EBUS scopes have enabled mapping of the elasticity of LNs in the form of colors and strain ratio leading to better guidance while taking samples [3].

Elastography is an ultrasonography-associated technology that measures tissue compressibility. and malignant in 16 (55.14\%) cases. On classifying type 1 group and type 2 group as 'benign' and type 3 group as malignant in nature, the sensitivity, specificity, negative predictive value, positive predictive value, and diagnostic accuracy rates were found to be $83.54,61.54,86.84,55.17$, and $78.10 \%$.

Conclusions The addition of elastography while performing EBUS of mediastinal lymphadenopathy is a technique that may be helpful in selecting sites for EBUS-guided transbronchial needle aspiration; however, more studies are needed to access its practical usefulness.

Egypt J Bronchol 2019 13:630-635

(C) 2020 Egyptian Journal of Bronchology

Egyptian Journal of Bronchology 2019 13:630-635

Keywords: elastography, endobronchial ultrasound, mediastinal lymphadenopathy

Max Super Specialty Hospital, Vaishali, Ghaziabad, Uttar Pradesh, India

Correspondence to Dr. Ankit Bhatia, MD, EDRM, IDCCM, W-3, Near

Radisson Blu Hotel, Sector-1, Vaishali, Ghaziabad, Uttar Pradesh, India. Pin code: 201012. Tel: +91 9711550306; fax: +911204173010;

e-mail: ankitbhatia85@gmail.com

Received: 5 June 2019 Accepted: 17 September 2019 Published: 21 January 2020

Endobronchial elastography can be performed in real time and the elastic responses to external compression may be converted to a color format of red, green, and blue images to allow for qualitative assessment of the soft tissue concerned.

We aim to discuss the potential role of endobronchial elastography in identifying LN features for better characterization of $\mathrm{LNs}$ and subsequently better selection of biopsies.

\section{Patients and methods}

This study was performed retrospectively on consecutive patients who were admitted for EBUStransbronchial needle aspiration (TBNA) at our department for a period of 6 months. Retrospective study done, all necessary consents obtained. The

This is an open access journal, and articles are distributed under the terms of the Creative Commons Attribution-NonCommercial-ShareAlike 4.0 License, which allows others to remix, tweak, and build upon the work non-commercially, as long as appropriate credit is given and the new creations are licensed under the identical terms. 
elastography evaluation was done in addition of the EBUS-TBNA for clinical indications independent of the purpose of the study. EBUS-TBNA was done for patients with mediastinal lymphadenopathy, taken as an enlargement of LNs of more than or equal to $1 \mathrm{~cm}$ in short-axis diameter on the computed tomography chest. The patients gave written informed consent before the EBUS-TBNA and to use records for the study. Patient confidentiality was maintained throughout the study.

All procedures were executed by the same physician. The procedure was performed on conscious sedation with midazolam and fentanyl with the addition of local lidocaine sprays. The EBUS convex probe (CPEBUS, BF-UC260FW; Olympus, Tokyo, Japan) was introduced through the oral route, with spray-as-you-go instillation of $2 \mathrm{ml}$ aliquot doses of $2 \%$ lidocaine.

Elastography was performed on mediastinal LNs that were seen to be candidates for EBUS-TBNA. The colors correlated with soft, firm, and hard tissues were yellow/red, green, and blue, respectively. The B-mode images and elastography images were displayed simultaneously on the screen alongside. Elastography evaluation was done on every node sampled. The predominant color was documented as interpreted by two independent bronchoscopists after the images were obtained. Subsequently, classification of the EBUS elastography images was done using the classification protocol used by Izumo et al. [4].

Type 1 group: largely nonblue (green, yellow, and red). Type 2 group: part blue, part nonblue (green, yellow, and red). Type 3 group: largely blue.

The final result was confirmed from the histopathological examination of EBUS-TBNA specimens made as cell blocks by pathologists who were not aware of the results of EBUS elastography. Subsequently, elastography color patterns were correlated with biopsy results.

\section{Statistical analysis}

The statistical analysis was carried out using SPSS software (18.0; SPSS Inc., Chicago, Illinois, USA). The statistical significance between groups was decided by $\chi^{2}$ test.

\section{Results}

A total of 80 patients were included in the study while 105 LNs were studied.

The patients' demographics and characteristics are given in Table 1.
Table 1 Patients' demographics and characteristics

\begin{tabular}{lc}
\hline No of patients & 80 \\
Age (years) & $43.73 \pm 14.93$ \\
Males & 55 \\
Females & 25 \\
LNs examined & 105 \\
LN stations & \\
Station 4L & 2 \\
Station 4R & 13 \\
Station 7 & 65 \\
Station 10R & 25 \\
Size & \\
$<20$ mm & 60 \\
$>20$ mm & 45 \\
Benign - tubercular & 52 \\
Benign - sarcoidosis & 27 \\
\hline
\end{tabular}

LN, lymph node.

Table 2 Elastographic patterns and colors compared with final pathologic diagnosis

\begin{tabular}{lcc}
\hline $\begin{array}{l}\text { Elastography } \\
\text { type }\end{array}$ & $\begin{array}{c}\text { Number of benign } \\
\text { LNs/total number } \\
{[n / N(\%)]}\end{array}$ & $\begin{array}{c}\text { Number of malignant } \\
\text { LNs/total number } \\
{[n / N(\%)]}\end{array}$ \\
\hline $\begin{array}{l}\text { Type 1 } \\
(N=46)\end{array}$ & $42 / 46(91.30)$ & $4 / 46(8.70)$ \\
Type 2 & & \\
$(N=30)$ & $24 / 30(80)$ & $6 / 30(20)$ \\
$\begin{array}{l}\text { Type 3 } \\
(N=29)\end{array}$ & $13 / 29(44.82)$ & $16 / 29(55.14)$ \\
\hline
\end{tabular}

LN, lymph node.

A qualitative classification based on the patterns and colors of LNs was performed. Elastographic patterns/colors were described according to the dominant colors and their distribution within the target LN.

The following representation formed the premise for the classification of elastographic types:

Type 1 group: largely nonblue (green, yellow, and red). Type 2 group: part blue, part nonblue (green, yellow, and red). Type 3 group: largely blue.

The elastography colors and patterns were compared with the eventual pathologic diagnosis (Table 2).

The nodes which were labeled as type 1 on EBUS elastography were benign in $42 / 46(91.30 \%)$ and malignant in $4 / 46(8.70 \%) ; 40 / 46$ were of size less than $20 \mathrm{~mm}$; for type $2 \mathrm{LNs}, 24 / 30$ (80\%) were benign and 6/20 (20\%) were malignant; 10/ 30 were of size less than $20 \mathrm{~mm}$; type 3 nodes were found to be of benign pathology in 13/29 (44.82\%) and malignant in 16 (55.14\%), 4/29 were of size less than $20 \mathrm{~mm}$. 
Figure 1

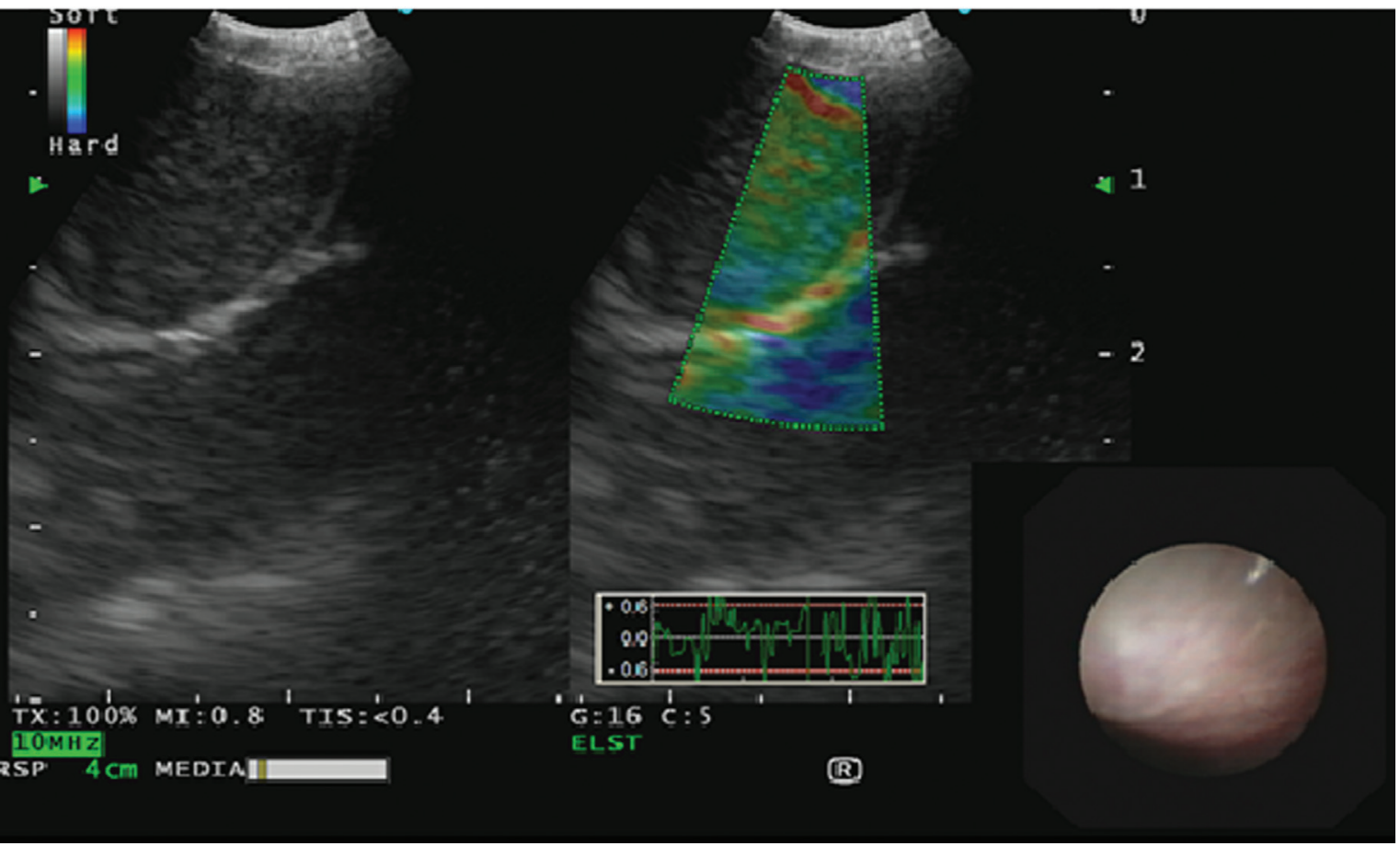

Type 1 can be seen on the elastography image.

Figure 2

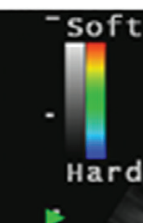

Tंx $: 100$ \% MI $: 0.8$ 'TIS: $<0.4$ $10 \mathrm{MHZ}$

RSP $4 \mathrm{Cm}$ MEDIAII

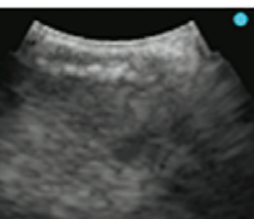

$\bullet$
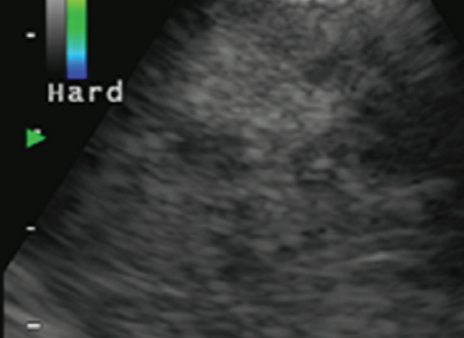

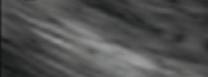

$=$

Type 2 can be seen on the elastography image.

On labeling type 1 and type 2 as 'benign,' and type 3 as 'malignant,' the sensitivity, specificity, positive predictive value, negative predictive value, and diagnostic accuracy rates were 83.54, 61.54,
86.84, 55.17, and 78.10\%, respectively. Only LNs with adequate lymphocytes or those with a definitive diagnosis were included for data analysis (Figs 1-3). 


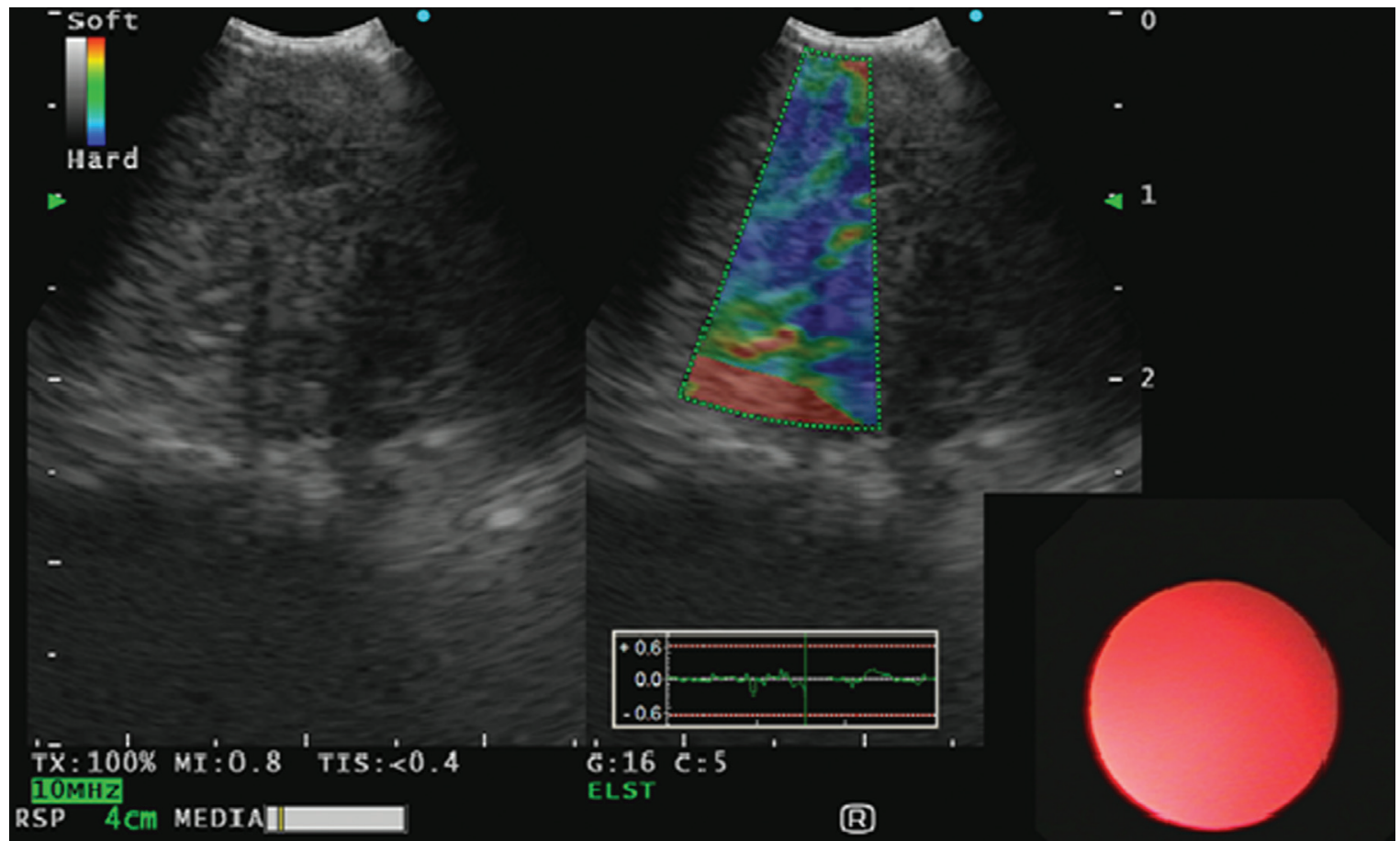

Type 3 can be seen on the elastography image.

\section{Discussion}

EBUS elastography complements the conventional EBUS without significant prolongation of procedure, cost, or added complications. It can be performed in real time during the procedure and can give important information that can impact the final diagnosis.

Elastography can be considered an imaging equivalent of the ancient skill of palpation. It is based on the principle that softer parts of tissues can deform easier under compression than the harder parts, allowing an objective and quantified determination of tissue consistency, showing differences in hardness between a diseased and normal tissue $[5,6]$.

A cancerous lesion is usually hard and dense than the surrounding tissues, with comparatively less deformation under the surrounding pressures of oppression. Elastography can reflect the elastic properties of tissues and hence can judge the relative hardness of the lesion, facilitating the characterization and diagnosis of mediastinal lesions [7].

Our sample size had more benign lesions as we have a higher prevalence of tubercular and sarcoid mediastinal LN enlargement in this part of the world contrary to Western data.
Elastography can be divided into two major divisions: qualitative one is based on the tissue response to an internal or external generated force, called strain elastography; quantitative one, based on measuring shear waves generated by a 'push-pulse' of low frequency, called shear wave elastography $[8,9]$.

Strain ration is a way to semiquantify information from strain images and can be calculated by measuring tissue stiffness in a targeted area comparing with an area outside of a region representing the normal tissue. The strain ratio value can then be calculated as the quotient between the two regions [10]. It is a semiquantitative method and has been found to give favorable results that have been reported for mediastinal LN analysis in patients with esophageal cancer.

This technology was initially used in the field of breast ultrasound and for evaluation of the thyroid, prostate, and liver [11-14].

Trosini-Désert et al. [15] in a small sample size, for the first time, showed that EBUS elastography was feasible and that the bronchial tracheal cartilage did not affect the collection of elastic data and concluded that elastography improved the diagnostic ability of EBUS.

One of the first initial reports on the use of EBUS elastography is by Izumo and colleagues, who analyzed 
75 LNs and classified the findings according to the color distribution: the nodes were classified in the same manner as in our study. On an evaluation of the LNs, 42 were malignant, 33 were found to be benign. The nodes that were classified as type 1 were benign by $100 \%$; for type $2 \mathrm{LNs}, 46.9 \%$ were found to be benign and $57.1 \%$ were malignant; and for type $3 \mathrm{LNs}, 5.4 \%$ were benign (2/37) and 94.6\% were malignant [4].

We had deviated results which could be accounted to more benign population in the study which led to increased fibrotic components in LNs which can cause ambiguity for the operator.

EBUS elastographic analysis can assist in guiding the puncture site in a non-necrotic part of the suspicious LN and hence improving the diagnostic accuracy and quality for further diagnostic testing.

Mean stiff ratios can also be calculated and used in analysis as was done by Nakajima et al. [16]. The group evaluated 49 LNs (16 malignant) in 21 patients by EBUS elastography. Mean stiff area ratios were found to be significantly high for the malignant nodes (0.478) than for benign LNs $(0.216 ; P=0.0002)$. The group was able to show a sensitivity and specificity for predicting malignant disease of 0.81 and 0.85 using a cutoff value of 0.311 for stiff area ratio. The group did not use any color distribution for an additional analysis.

In a group of 40 lung cancer patients, a Chinese group, $\mathrm{He}$ and colleagues evaluated in a group of 40 lung cancer patients, the accuracy of B-mode features, elastographic strain ratio and a qualitative elastographic score to predict metastatic LN involvement. The elastographic score proved to have more sensitivity and specificity in determining the malignant $\mathrm{LN}$ s than all $\mathrm{B}$-mode EBUS criteria. Further, the combination of B-mode criteria and elastography improved the diagnostic accuracy of EBUS to differentiate between benign and metastatic mediastinal LNs [17].

However, EBUS elastography is not meant currently to replace EBUS-fine needle aspiration for mediastinal LN diagnosis but it may be useful as a supplemental method to reduce the number of punctures and improve the yield of fine needle aspiration [18].

The potential application for elastography in EBUS would be targeting higher risk areas within normal looking sized LNs. It can assist in guiding the puncture site in the non-necrotic part of a suspicious LN when the necrotic tissue is present, as seen in advanced cancers. The real challenge would be in the evaluation of benign lesions where the fibrotic component would be a hindrance to a good mapping quality.

It can become an important tool in selecting nodes for sampling, thus improving the overall yield.

Several limitations should be highlighted in the present study. First, the number of LNs studied was limited. Second, previous studies suggested that strain ratio of elastography may be an objective indicator for the classification of LNs; however, we did not take this feature as we wanted a fast and practical way of predicting the characteristic of the LN.

\section{Conclusion}

In conclusion, our study shows a significant practical clinical utility of the addition of elastography mode for classifying and guiding TBNA in suspicious mediastinal LNs with a high positive predictive value and diagnostic accuracy.

\section{Financial support and sponsorship \\ Nil.}

\section{Conflicts of interest}

There are no conflicts of interest.

\section{References}

1 Seides BJ, Egan JP, French KD, Kovitz KL, Desai NR. Fiducial marker placement for stereotactic body radiation therapy via convex probe endobronchial ultrasound: a case series and review of literature. $J$ Thorac Dis 2018; 10:1972-1983.

2 Sircar M, Bhatia A, Gupta R, Kaur R. Bronchoscopic decompression of mediastinal lymph node abscess using endobronchial ultrasound. J Pulm Respir Med 2016; 6:315.

3 Joshi S, Gupta R, Bhatia A, Ali SJ. The sonoelastographic characters of noncomplicated bronchogenic cyst: an evaluation using CP-EBUS. $J$ Assoc Chest Physicians 2018; 6:41-43.

4 Izumo T, Sasada S, Chavez C, Matsumoto Y, Tsuchida T. Endobronchial ultrasound elastography in the diagnosis of mediastinal and hilar lymph nodes. Jpn J Clin Oncol 2014; 44:956-962.

5 Emerson K. Diseases of the breast. In: Thorn WW, ed. Principals of internal medicine. 7 th ed. New York: McGraw-Hill 1974. 582-587

6 Bhargava S, Bhargava SK, Sharma S, Prakash M. Elastography: a new imaging technique and its application. JIMSA 2013; 26:25-30.

7 Li P, Zheng W, Zhao L. Convex probe endobronchial ultrasound: applications beyond conventional indications. J Thorac Dis 2015; 7: E289-E297.

8 Barr RG. U S elastography: applications in tumors. In: Luna A, ed. Functional imaging in oncology. Berlin Heidelberg: Springer-Verlag 2014. 459-488

9 Dietrich CF. Elastography applications. Endo Heute 2011; 24:177-212.

10 Rana SS, Vilmann P. Endoscopic ultrasound features of chronic pancreatitis: a pictorial review. Endosc Ultrasound 2015; 4:10-14.

11 Garra BS, Cespedes El, Ophir J, Spratt SR, Zuurbier RA, Magnant CM, Pennanen MF. Elastography of breast lesions: initial clinical results. Radiology 1997; 202:79-86.

12 Lyshchik A, Higashi T, Asato R, et al. Thyroid gland tumor diagnosis at US elastography. Radiology 2005; 237:202-211.

13 König K, Scheipers U, Pesavento A, et al. Initial experiences with real-time elastography guided biopsies of the prostate. J Urol 2005; 174:115-117. 
14 Foucher J, Chanteloup E, Vergniol J, et al. Diagnosis of cirrhosis by transient elastography (FibroScan): a prospective study. Gut 2006; 55:403-408.

15 Trosini-Désert V, Jeny F, Taillade L, Vignot S, ZribiH, Capron F, Similowski T. Bronchial endoscopic ultrasound elastography: preliminary feasibility data. Eur Respir J 2013; 41:477-479.

16 Nakajima $T$, Inage $T$, Sata $Y$, et al. Elastography for predicting and localizing nodal metastases during endobronchial ultrasound. Respiration 2015; 90:499-506.
$17 \mathrm{He} \mathrm{H}$, Lu X, Ma H, Zhu J, Huang M. Value of endobronchial ultrasound elastography in the diagnosis of mediastinal and hilar lymph node metastasis in lung cancer. Zhong Nan Da Xue Xue Bao Yi Xue Ban 2016; 41:30-36.

18 Xu W, Shi J, Zeng X, Li X, Xie WF, Guo J, Lin Y. EUS elastography for the differentiation of benign and malignant lymph nodes: a meta-analysis. Gastrointest Endosc 2011; 74 : 1001-1009. 